\title{
The eccentricity distribution of compact binaries
}

\author{
I. Kowalska ${ }^{1}$, T. Bulik ${ }^{1,2}$, K. Belczynski ${ }^{1,3}$, M. Dominik ${ }^{1}$, and D. Gondek-Rosinska ${ }^{4,2}$ \\ 1 Astronomical Observatory, University of Warsaw, Al Ujazdowskie 4, 00-478 Warsaw, Poland \\ e-mail: ikowalska@astrouw.edu.pl \\ 2 Nicolaus Copernicus Astronomical Center, Bartycka 18, 00716 Warsaw, Poland \\ 3 Dept. of Physics and Astronomy, University of Texas, Brownsville, TX 78520, USA \\ ${ }^{4}$ Institute of Astronomy, University of Zielona Góra, ul. Lubuska 2, 65-265 Zielona Góra, Poland
}

Received 17 September 2010 / Accepted 9 December 2010

\begin{abstract}
Context. The current gravitational wave detectors have reached their operational sensitivity and are nearing detection of compact object binaries. In the coming years, we expect that the Advanced LIGO/VIRGO will start taking data. At the same time, there are plans for third generation ground-based detectors such as the Einstein Telescope, and space detectors such as DECIGO.

Aims. We discuss the eccentricity distribution of inspiral compact object binaries during they inspiral phase. We analyze the expected distributions of eccentricities at three frequencies that are characteristic of three future detectors: Advanced LIGO/VIRGO (30 Hz), Einstein Telescope $(3 \mathrm{~Hz})$, and DECIGO $(0.3 \mathrm{~Hz})$.

Methods. We use the StarTrack binary population code to investigate the properties of the population of compact binaries in formation. We evolve their orbits until the point that they enter a given detector sensitivity window and analyze the eccentricity distribution at that time.

Results. We find that the eccentricities of $\mathrm{BH}-\mathrm{BH}$ and $\mathrm{BH}-\mathrm{NS}$ binaries are quite small when entering the Advanced LIGO/VIRGO detector window for all considered models of binary evolution. Even in the case of the DECIGO detector, the typical eccentricities of $\mathrm{BH}-\mathrm{BH}$ binaries are below $10^{-4}$, and the BH-NS eccentricities are smaller than $10^{-3}$. Some fraction of NS-NS binaries may have significant eccentricities. Within the range of considered models, we found that a fraction of between $0.2 \%$ and $2 \%$ NS-NS binaries will have an eccentricity above 0.01 for the Advanced LIGO/VIRGO detectors. For the ET detector, this fraction is between $0.4 \%$ and $4 \%$, and for the DECIGO detector it lies between $2 \%$ and $27 \%$.
\end{abstract}

Key words. binaries: general - gravitational waves

\section{Introduction}

As the interferometric gravitational wave detectors LIGO and VIRGO (Harry and the LIGO Scientific Collaboration 2010; Acernese et al. 2006) reach their design sensitivities, the first detection of gravitational waves has become more imminent. Both detectors will undergo serious improvements to increase their sensitivity (Smith and LIGO Scientific Collaboration 2009; Spallicci et al. 2005).

It is therefore important to investigate the properties of the primary candidate sources for detection, namely compact object binaries. There have been a number of papers dealing with several properties of the population of compact binaries (e.g., Nelemans \& van den Heuvel 2001; Voss \& Tauris 2003; De Donder \& Vanbeveren 2004; Sipior \& Sigurdsson 2002; Pfahl et al. 2005; Dewi et al. 2002, 2005; Bogomazov et al. 2007; Kiel et al. 2010). In particular, Abadie et al. (2010) presented their estimated detection rates. In addition, the mass spectrum (Gondek-Rosińska et al. 2007), and even spin properties (Schnittman 2004; Mandel \& O'Shaughnessy 2010) have been studied. In this paper, we present yet another aspect of the merging compact object binary population: the distribution of the eccentricity.

From radio observations, we currently know of only six compact object binaries with merger timescales shorter than a Hubble time, all of them NS-NS (neutron star - neutron star) systems, and we know of no BH-NS (black hole - neutron star) nor BH-BH (black hole - black hole) system. The known NS-NS systems are listed along with their orbital parameters in Table 1.

The observed NS-NS binaries have merger times $T_{\text {merg }} \gtrsim 100$ Myr. However, a significant fraction of the population of the merging NS-NS may originate in the so-called ultra-compact NS-NS binaries, which have much shorter merger timescales $T_{\text {merg }} \lesssim 100$ Myr (Belczynski et al. 2002a). In addition, the small number of known pulsars are indicative of there being a significant fraction of very eccentric binaries.

Since no BH-NS nor BH-BH binaries are known, we can only rely on evolutionary considerations when estimating their number and properties. It has been found that their number depends very strongly on the outcome of the common envelope phase when the secondary is on the Hertzsprung gap. This phase will very likely end up as a merger and the formation of a Thorne-Zytkow object. However, it has been demonstrated that in a low-metallicity environment the common envelope mergers may be (to some extent) avoided and the $\mathrm{BH}-\mathrm{BH}$ formation is very effective (Belczynski et al. 2010b).

The eccentricity of a compact object binary may potentially be derived by analyzing the inspiral signal, provided that the eccentricity is significant. In this paper, we investigate the eccentricity distributions in the frequency band of the currently working and future detectors of gravitational waves. For the currently working detectors (LIGO and VIRGO), we assume that the sensitivity of the detectors will allow us to measure the signal for the frequencies starting at $30 \mathrm{~Hz}$. This may not be 
Table 1. Known merging compact object binaries.

\begin{tabular}{cccccccl}
\hline \hline Name & $P_{\text {orb }}[h]$ & Present $e$ & $T_{\text {merge }}[\mathrm{Gyr}]$ & $e$ at $0.3 \mathrm{~Hz}$ & $e$ at $3 \mathrm{~Hz}$ & $e$ at $30 \mathrm{~Hz}$ & Ref. \\
\hline J0737-3039A/B & 2.454 & 0.088 & 0.085 & $4.5 \times 10^{-5}$ & $4 \times 10^{-6}$ & $3.5 \times 10^{-7}$ & Burgay et al. (2003) \\
B2127+11C & 8.05 & 0.681 & 0.2 & $2.9 \times 10^{-4}$ & $2.6 \times 10^{-5}$ & $2.3 \times 10^{-6}$ & Anderson et al. (1990) \\
J1906+0746 & 3.98 & 0.085 & 0.3 & $2.6 \times 10^{-5}$ & $2.3 \times 10^{-6}$ & $2 \times 10^{-7}$ & Lorimer et al. (2006) \\
B1913+16 & 7.752 & 0.617 & 0.3 & $2.2 \times 10^{-4}$ & $1.9 \times 10^{-5}$ & $1.7 \times 10^{-6}$ & Weisberg \& Taylor (2005) \\
J1756-2251 & 7.67 & 0.181 & 1.7 & $2.6 \times 10^{-5}$ & $2.5 \times 10^{-6}$ & $2.2 \times 10^{-7}$ & Faulkner et al. (2005) \\
B1534+12 (=J1537+1155) & 10.098 & 0.274 & 2.7 & $3.6 \times 10^{-5}$ & $3.2 \times 10^{-6}$ & $2.8 \times 10^{-7}$ & Wolszczan (1991) \\
\hline
\end{tabular}

accurate for the current state of these instruments, but it does accurately represent the predicted sensitivity of the Advanced LIGO/VIRGO detectors. We consider two future detectors: the Einstein Telescope (Van Den Broeck 2010) and DECIGO (Kawamura 2006; Seto et al. 2001). For the Einstein Telescope, we assume that binaries shall be detectable from $3 \mathrm{~Hz}$, and for DECIGO we assume that the lowest frequency detectable is $0.3 \mathrm{~Hz}$. In all cases, these have to be treated as indicative numbers that roughly describe these instruments.

In Sect. 2, we describe the model used to investigate the population of compact object binaries. Section 3 presents the results for the current and future gravitational wave detectors. In Sect. 4, we summarize and discuss the results.

\section{The model}

\subsection{Compact object binary population model}

To model the population of compact object binaries, we used the StarTrack population synthesis code (Belczynski et al. 2002b). It perform a suite of Monte Carlo simulations of the stellar evolution of stars in environments of two typical metallicities: $Z=Z_{\odot}=0.02$ and $Z=10 \% Z_{\odot}=0.002$ (e.g., Belczynski et al. 2010b). In these calculations, we employed the recent estimates of mass loss rates (Belczynski et al. 2010a). We calculate a population of 2 million massive binary stars, tracking the ensuing formation of relativistic binary compact objects: double neutron stars (NS-NS), double black hole binaries (BH-BH), and mixed systems (BH-NS). Our modeling utilizes updated stellar and binary physics, including results from supernova simulations (Fryer \& Kalogera 2001) and compact object formation (Timmes et al. 1996), incorporating elaborate mechanisms for treating stellar interactions such as mass transfer episodes (Belczynski et al. 2008) or tidal synchronization and circularization (Hut 1981). We place special emphasis on the common envelope evolution phase (Webbink 1984), which is crucial for close double compact object formation because the attendant mass transfer permits an efficient hardening of the binary. This orbital contraction can be sufficiently efficient to cause the individual stars in the binary to coalesce and form a single highly rotating object, thereby preventing additional binary evolution and the formation of a double compact object. Because of significant radial expansion, stars crossing the Hertzsprung gap (HG) very frequently initiate a common envelope phase. HG stars do not have a clear entropy jump at the core-envelope transition (Ivanova \& Taam 2004); if such a star overflows its Roche lobe and initiates a common envelope phase, the inspiral is expected to lead to a coalescence (Taam \& Sandquist 2000). In particular, it has been estimated that for a solar metallicity environment (e.g., our Galaxy), properly accounting for the HG gap may lead to a reduction in the merger rates of $\mathrm{BH}-\mathrm{BH}$ binaries by $\sim 2-$ 3 orders of magnitude (Belczynski et al. 2007). In contrast, in a low metallicity environment this suppression is much less severe
Table 2. The list of models of stellar evolution used in the paper.

\begin{tabular}{cccc}
\hline \hline Model & Metallicity & $\sigma\left[\mathrm{km} \mathrm{s}^{-1}\right]$ & $\mathrm{HG}$ \\
\hline AZK & $Z_{\odot}$ & 265.0 & + \\
BZK & $Z_{\odot}$ & 265.0 & - \\
AZk & $Z_{\odot}$ & 132.5 & + \\
BZk & $Z_{\odot}$ & 132.5 & - \\
AzK & $10 \% Z_{\odot}$ & 265.0 & + \\
BzK & $10 \% Z_{\odot}$ & 265.0 & - \\
Azk & $10 \% Z_{\odot}$ & 132.5 & + \\
Bzk & $10 \% Z_{\odot}$ & 132.5 & - \\
\hline
\end{tabular}

( $\sim 1$ order of magnitude; Belczynski et al. 2010b). The details of the common envelope phase are not yet fully understood, thus in what follows we consider two set of models, one that does not take into account the suppression (optimistic models: marked with $\mathrm{A}$ ), and another that assumes the maximum suppression (pessimistic models: marked with B). Solar metallicity and $10 \%$ of solar metallicity are labeled with $Z$ and $z$, respectively. In the case of NSs, we adopt natal kick distributions from observations of single Galactic pulsars (Hobbs et al. 2005) with $\sigma=265 \mathrm{~km} \mathrm{~s}^{-1}$. However, for BHs we draw kicks from the same distribution (but at a lower magnitude), which is inverse proportional to the amount of fall back expected at BH formation (e.g., Fryer \& Kalogera 2001). In particular, for most massive BHs that form with the full fall back (direct BH formation), the amount of natal kick is zero. In addition, we test one more set of models in which the magnitude of the NS kicks is lower by a factor of 2, to $\sigma=132.5 \mathrm{~km} \mathrm{~s}^{-1}$, as some observations and empirically based arguments seem to indicate that natal kicks in close binaries are lower than for single stars (Dessart et al. 2006; Kitaura et al. 2006). The BH kicks are decreased in the similar fashion as in models with the full NS kicks. The standard value of $\sigma$ parameter is denoted by $K$ and the smaller value by $k$. The detailed list of models considered in this paper is presented in Table 2. Model AZK is a standard set of parameters described in detail by Belczynski et al. (2002b).

\subsection{Evolution of orbits}

The evolution of the orbit of compact object binary under the influence of gravitational radiation had been calculated by Peters \& Mathews (1963); Peters (1964). In the quadrupole approximation, the orbit decays as

$$
\frac{\mathrm{d} a}{\mathrm{~d} t}=-\frac{\beta}{a^{3}} \Psi(e), \quad \Psi(e)=\frac{1+73 / 24 e^{2}+37 / 96 e^{4}}{\left(1-e^{2}\right)^{7 / 2}},
$$

where $a$ is the great semi-axis, $e$ is the eccentricity of binary, $M_{1}$ is the mass of the first component, $M_{2}$ is the mass of second component, and

$$
\beta=\frac{64}{5} \frac{G^{3} \mu M^{2}}{c^{5}}, \quad \mu=\frac{M_{1} M_{2}}{M_{1}+M_{2}} .
$$




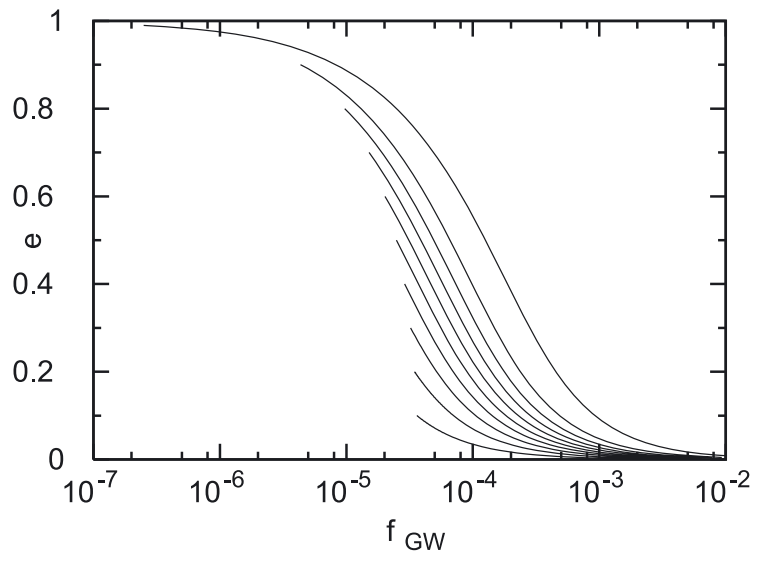

Fig. 1. We present ten cases of eccentricity evolution, starting with different values of e from $e=0.1$ (first line from the bottom) to $e=0.99$ (first line from the top). Initial semi-major axis is chosen such that a binary will merge within time $T_{\text {merg }}=10 \mathrm{Gyr}$ in each case.

While the eccentricity decays as

$$
\frac{\mathrm{d} e}{\mathrm{~d} t}=-\frac{19}{12} \frac{\beta}{a^{4}} \Phi(e), \quad \Phi(e)=\frac{\left(1+121 / 304 e^{2}\right) e}{\left(1-e^{2}\right)^{5 / 2}} .
$$

Using the above formulae we can express the fundamental gravitational wave frequency as a function of the eccentricity

$$
f_{\mathrm{GW}}(e)=\frac{2}{P_{0}} \frac{\left(1-e^{2}\right)^{3 / 2}}{e^{18 / 19}}\left[1+\frac{121}{304} e^{2}\right]^{-1305 / 2299} c_{0}^{3 / 2},
$$

where $c_{0}=\left(e_{0}^{12 / 19}\left[1+\frac{121}{304} e_{0}^{2}\right]^{1305 / 2299}\right)\left(1-e_{0}^{2}\right)^{-1}, P_{0}$ is the initial orbital period, and $f_{\mathrm{GW}}(e)$ is the first non-zero harmonic. The gravitational wave frequency is twice the orbital frequency, i.e., $f_{\mathrm{GW}}=2 f_{\text {orb }}=\frac{2}{P_{\text {orb }}}$.

We present the evolution of eccentricity as a function of gravitational wave frequency in Fig. 1 for a binary neutron star with components of equal masses of $1.4 M_{\odot}$. The initial frequency corresponds to a semi-major axis such that the merger time is set to be $T_{\text {merg }}=10^{4} \mathrm{Myr}$. Figure 1 contains several different cases of evolution in the plane stretched by eccentricity and gravitational wave frequency.

\section{Results}

\subsection{Properties of the binaries at formation time}

We start with an initial population created using the StarTrack code. We present the properties of the population of compact object binaries in Figs. 2-4 in the space spanned by the initial eccentricity and initial gravitational wave frequency, which is twice the orbital frequency. Each panel in theses figures corresponds to a different model labeled as listed in Table 2.

The case of the NS-NS systems is shown in Fig. 2. The boundary of the region populated by the systems on the left-hand side corresponds to the requirement that we only consider binaries that merge within a Hubble time. The bulk of the binaries shown in each panel correspond to those that have undergone one CE phase in their evolution. The top row corresponds to the models AZK, AZk, AzK, and Azk, in which we allow the binaries to cross through the common envelope with the donor on the Hertzsprung gap, denoted by "+" in Table 2. These binaries may undergo a second common envelope phase with a helium star companion. At the second CE stage, the orbit is tightened even more leading to formation of the stripe in the diagram stretching from $f_{\mathrm{GW}} \approx 10^{-2} \mathrm{~Hz}$ at $e \approx 10^{-2}$. In these models, the initial distribution in the space of gravitational wave frequency versus eccentricity is bimodal. The influence of the value of the kick velocity has a small impact on the shape of distributions presented in Fig. 2 as can be seen by comparing the data in plots labeled as either K-large kicks or k-small kicks.

For BH-NS systems, presented in Fig. 3, and BH-BH binaries, in Fig. 4, we present the results of six out of eight models, since in models BZK and BZk, almost no binaries are formed in our simulations that involve $2 \times 10^{6}$ initial binaries. For BHNS and $\mathrm{BH}-\mathrm{BH}$ binaries, the formation of ultra-compact binaries is not expected. The formation of NS-NS ultra-compact systems in very close orbits is the consequence of the final CE episode, which is initiated by a low-mass helium (2-4 $\left.M_{\odot}\right)$ star and its NS companion $\left(1.4 M_{\odot}\right)$. Since the donor is about twice as massive as its companion, the CE phase is initiated by the non-stable mass transfer and the orbit significantly decreased in size. For more massive $\mathrm{BH}-\mathrm{BH} / \mathrm{BH}-\mathrm{NS}$ binaries, helium stars are on average more massive $\left(M>3-4 M_{\odot}\right)$ and do not expand (so no CE phase), and even if a low mass helium star forms, then its companion is a $\mathrm{BH}\left(M>3 M_{\odot}\right)$, so most likely instead of CE the RLOF is stable and does not lead to orbital decay (mass ratio $\sim 1$ ). Very few systems (e.g., models AzK or Azk) produce ultra-compact BH-NS/BH-BH binaries for very special cases of binary evolution. In the case of $\mathrm{BH}-\mathrm{BH}$ binaries, shown in Fig. 4 we present only six models, since models BZK and BZk do not lead to the formation of BH$\mathrm{BH}$ binaries (Belczynski et al. 2007). In all models, there is an enhanced density of systems formed with $e \approx 0.1$ at approximately $10^{-5} \mathrm{~Hz}<f_{\mathrm{GW}}<10^{-4} \mathrm{~Hz}$. In these systems, the second black hole has formed via direct collapse. When treating the direct collapse, we assume that $10 \%$ of the mass escapes in the form of neutrinos and possibly gravitational waves. Hence, the gravitational mass of the $\mathrm{BH}$ is $10 \%$ lower than the baryon mass of the collapsing star. This introduces a small eccentricity $\approx 0.1$ since the systems were circularized in the mass transfer prior to the collapse and the formation of the second $\mathrm{BH}$.

\subsection{Eccentricity when binary enters detector band}

For the detection of gravitational waves, it is important to know the eccentricity of a binary at the time it enters the sensitivity window of the detector. We consider three cases that correspond approximately to three types of detectors. In Fig. 5, we show the eccentricity distributions at $0.3 \mathrm{~Hz}$ (bottom horizontal axis) and $3 \mathrm{~Hz}$ (top horizontal axis) corresponding approximately to the ET and DECIGO detectors. The results for the Advanced LIGO/VIRGO can be easily obtained by rescaling the horizontal axis.

The shape of the eccentricity distributions at the moment that the binary enters the given detector band follows from the corresponding initial distribution. However, one must note that for each type of binary there is a different natural timescale and frequency, because of the different mass scales of each binary.

We present the results for the DECIGO detector and add appropriate numbers for the ET in parentheses. For the NS-NS binaries shown in the top panel of Fig. 5, the distribution is either centered on $e \approx 10^{-4}$ (ET: $10^{-5}$ ) for the models BZK, $\mathrm{BZk}, \mathrm{BzK}$, and Bzk, where we do not allow the formation of ultra-compact binaries in a second $\mathrm{CE}$ phase. The remaining models AZK, AZk, AzK, and Azk contain another component centered roughly at $e \approx 10^{-4}$ (ET: $10^{-3}$ ). This additional component represent the ultra-compact binaries that have experienced 

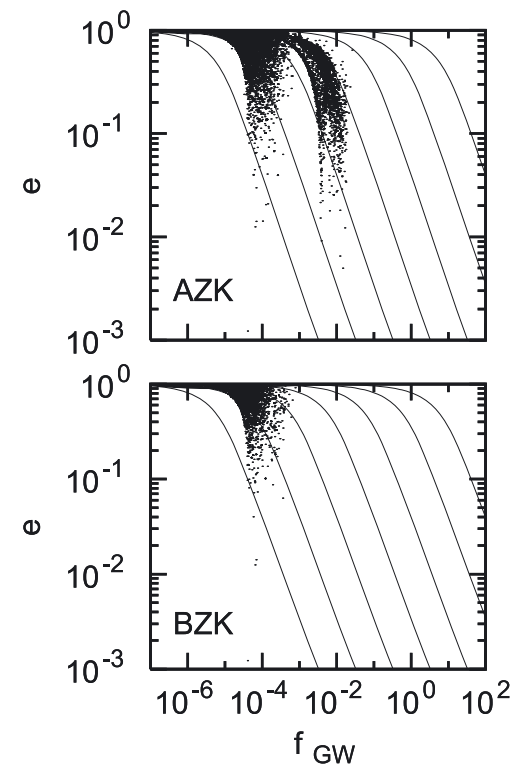
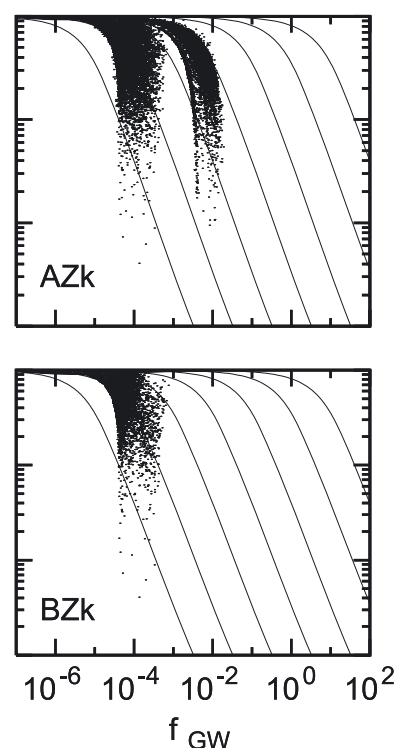
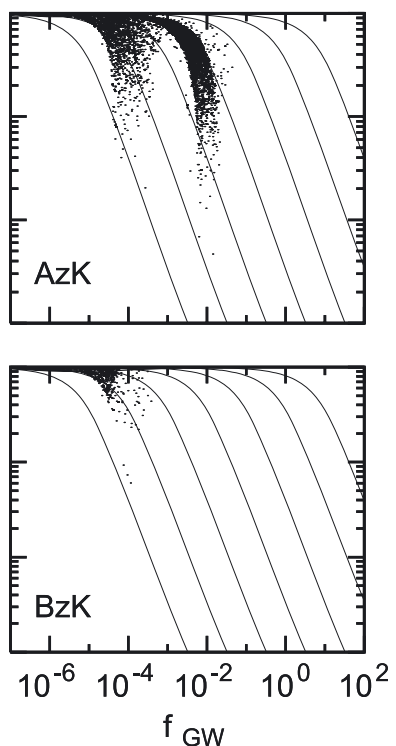
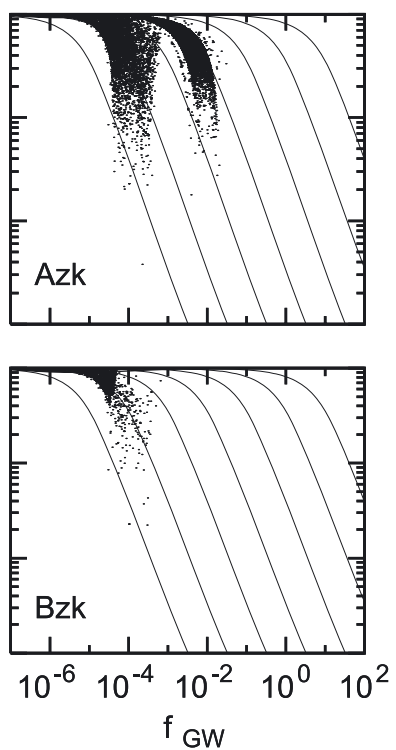

Fig. 2. The properties of the population of double neutron stars obtained using the StarTrack code. The plot shows only the binaries that will merge within the Hubble time. Solid lines correspond to evolutionary tracks for initial gravitational waves frequencies from $f_{0}=10^{-8} \mathrm{~Hz}$ (first line from the left-hand side) to $f_{0}=10^{2} \mathrm{~Hz}$ (first line from the right-hand side).
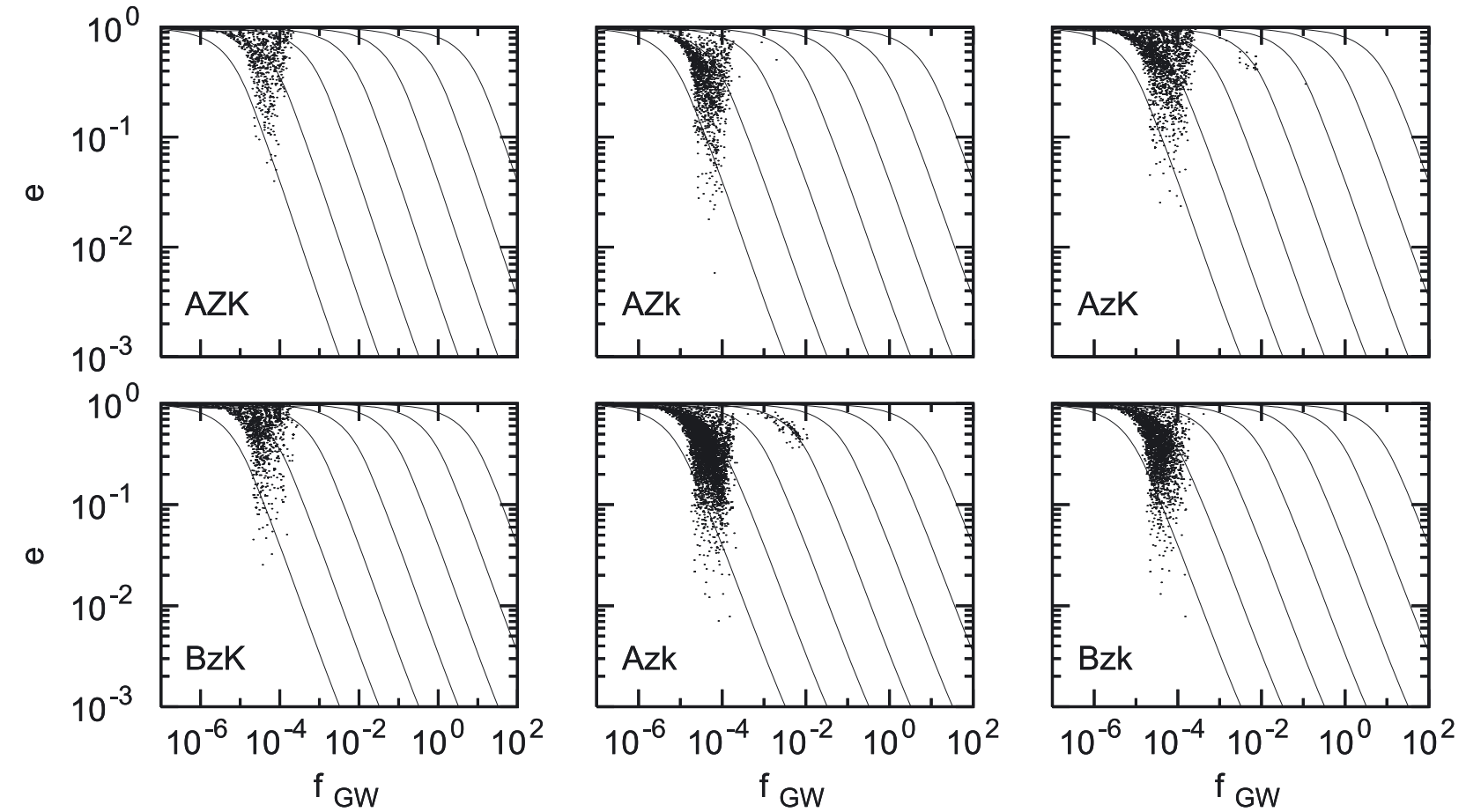

Fig. 3. The properties of the population of neutron star - black hole systems obtained using the StarTrack code. The plot shows only the binaries that will merge within the Hubble time. Solid lines correspond to evolutionary tracks for initial gravitational wave frequencies from $f_{0}=10^{-8} \mathrm{~Hz}$ (first line from the left-hand side) to $f_{0}=10^{2} \mathrm{~Hz}$ (first line from the right-hand side).

two episodes of mass transfer in their evolutionary history and were already very tight at the second supernova explosion. The mixed BH-NS binaries, shown in the middle panel of Fig. 5 exhibit a distribution of eccentricity centered at $e \approx 3 \times 10^{-5}$ (ET: $3 \times 10^{-6}$ ), while the eccentricity $\mathrm{BH}-\mathrm{BH}$ binaries, shown in the bottom panel of Fig. 5 lie between $e \approx \times 10^{-6}$ (ET: $10^{-7}$ ) and $e \approx \times 10^{-4}\left(\mathrm{ET}: 10^{-5}\right)$.

For the Advanced LIGO/VIRGO detectors where we assume that the low frequency boundary lies at $\approx 30 \mathrm{~Hz}$, the eccentricities are even smaller. It follows from Eq. (4) that the distributions are shifted by a factor of $10^{-19 / 18}$ for each factor of ten in frequency. Thus, the values of eccentricity in the case of Advanced LIGO/VIRGO type detectors are consistent with $e=0$ and we can safely assume that all BH-NS and BH-BH binaries are circular without any loss of sensitivity.

In Table 3, we present the fraction of binaries with eccentricities above 0.01 at the time of entering the detector band, to help quantify the extent of the large eccentricity tails of the distributions presented in Fig. 5. This fraction does not reflect the detectability of eccentricity (Shapiro Key \& Cornish 2010), 
I. Kowalska et al.: The eccentricity distribution of compact binaries
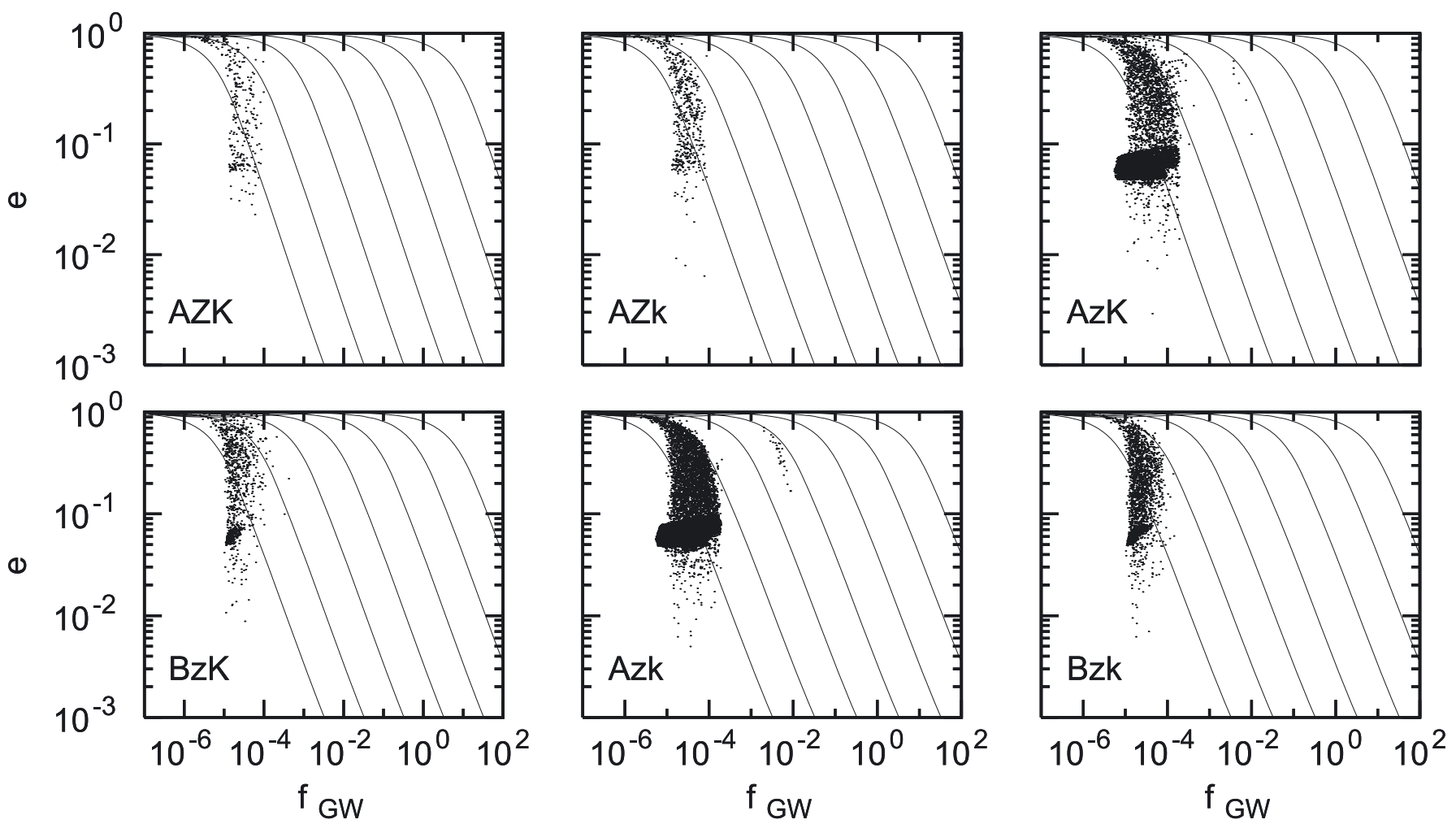

Fig. 4. The properties of the population of double black holes obtained using the StarTrack code. The plot shows only the binaries that will merge within the Hubble time. Solid lines correspond to evolutionary tracks for initial gravitational waves frequencies from $f_{0}=10^{-8} \mathrm{~Hz}($ first line from the left-hand side) to $f_{0}=10^{2} \mathrm{~Hz}$ (first line from the right-hand side).

Table 3. Fraction of the compact binaries with eccentricity greater than $10^{-2}$.

\begin{tabular}{|c|c|c|c|c|c|c|}
\hline \multicolumn{7}{|c|}{ NS-NS } \\
\hline & \multicolumn{2}{|l|}{$30 \mathrm{~Hz}$} & \multicolumn{2}{|c|}{$3 \mathrm{~Hz}$} & \multicolumn{2}{|c|}{$0.3 \mathrm{~Hz}$} \\
\hline AZK & $0.60 \%$ & (51) & $1.32 \%$ & (112) & $11.13 \%$ & $(945)$ \\
\hline BZK & $1.27 \%$ & (36) & $2.33 \%$ & $(66)$ & $6.52 \%$ & (185) \\
\hline AZk & $0.16 \%$ & (27) & $0.38 \%$ & (64) & $10.37 \%$ & (1732) \\
\hline BZk & $0.30 \%$ & (15) & $0.75 \%$ & (37) & $2.22 \%$ & $(110)$ \\
\hline $\mathrm{AzK}$ & $0.29 \%$ & (25) & $0.96 \%$ & (83) & $21.74 \%$ & (1880) \\
\hline BzK & $1.87 \%$ & (13) & $4.02 \%$ & (28) & $9.33 \%$ & (65) \\
\hline Azk & $0.26 \%$ & (37) & $0.57 \%$ & (81) & $26.91 \%$ & (3799) \\
\hline Bzk & $1.74 \%$ & (21) & $3.31 \%$ & (40) & $7.79 \%$ & (94) \\
\hline \multicolumn{7}{|c|}{ 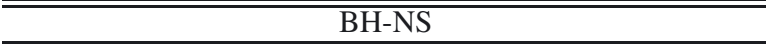 } \\
\hline & \multicolumn{2}{|c|}{$30 \mathrm{~Hz}$} & \multicolumn{2}{|c|}{$3 \mathrm{~Hz}$} & \multicolumn{2}{|c|}{$0.3 \mathrm{~Hz}$} \\
\hline AZK & $0.29 \%$ & (2) & $0.73 \%$ & (5) & $3.05 \%$ & (21) \\
\hline AZk & $0.15 \%$ & (2) & $0.54 \%$ & (7) & $0.61 \%$ & (8) \\
\hline AzK & $0.56 \%$ & (14) & $0.96 \%$ & (24) & $3.96 \%$ & (99) \\
\hline BzK & $0.68 \%$ & (10) & $1.23 \%$ & (18) & $3.63 \%$ & (53) \\
\hline Azk & $0.35 \%$ & (15) & $0.78 \%$ & (34) & $2.81 \%$ & (122) \\
\hline Bzk & $0.33 \%$ & (8) & $0.91 \%$ & (22) & $1.53 \%$ & (37) \\
\hline \multicolumn{7}{|c|}{$\overline{\text { BH-BH }}$} \\
\hline & \multicolumn{2}{|c|}{$30 \mathrm{~Hz}$} & \multicolumn{2}{|c|}{$3 \mathrm{~Hz}$} & \multicolumn{2}{|c|}{$0.3 \mathrm{~Hz}$} \\
\hline AZK & $0.31 \%$ & (1) & $0.62 \%$ & (2) & $1.87 \%$ & (6) \\
\hline AzK & $0.02 \%$ & (3) & $0.02 \%$ & (4) & $0.13 \%$ & (23) \\
\hline BzK & $0.15 \%$ & (2) & $0.15 \%$ & (2) & $0.46 \%$ & (6) \\
\hline Azk & $0.00 \%$ & (1) & $0.01 \%$ & (2) & $0.03 \%$ & (6) \\
\hline
\end{tabular}

Notes. Top table corresponds to double neutron stars (NS-NS), middle to the mixed systems (BH-NS), and the bottom to the binary black holes $(\mathrm{BH}-\mathrm{BH})$. We present the fraction of binaries at the moment of entering different frequency bands $(30 \mathrm{~Hz}, 3 \mathrm{~Hz}$, and $0.3 \mathrm{~Hz})$. In brackets, we include the number of these systems in the simulation $\mathrm{N}$. We only listed results for models that are non-zero. The number of digits shown is for formatting only, and the relative sampling error is $N^{-1 / 2}$. which for realistic distributions of binaries will be discussed in a forthcoming paper.

\section{Summary}

We have presented the eccentricity distributions of compact object binaries at three frequencies immediately before merger. The properties of the compact object binaries have been calculated using the StarTrack population synthesis code. We have found that the eccentricity distributions of the compact object binaries do not depend strongly on the assumed model of binary evolution. Any dependence has been found to be the strongest for binary neutron stars, whose distributions may be either single or double peaked. The extra peak corresponds to ultra-compact NS-NS binaries that have undergone an additional CE phase immediately before forming the second NS.

To make the results easier to use in the simulations, we have fitted the resulting distributions of eccentricity with a single lognormal distribution in the case of $\mathrm{BH}-\mathrm{BH}$ and $\mathrm{BH}-\mathrm{NS}$ binaries

$$
f(x)=\frac{1}{\sigma \sqrt{2 \pi}} \exp \left(-\frac{(x-\mu)^{2}}{2 \sigma^{2}}\right),
$$

where $x=\log e, \mu$ is the mean, and $\sigma$ is the variance.

For the NS-NS eccentricities, we used a sum of two lognormal distributions with two weights, since the distribution is double peaked, given by

$$
f(x)=\frac{w}{\sigma_{1} \sqrt{2 \pi}} \exp \left(-\frac{\left(x-\mu_{1}\right)^{2}}{2 \sigma_{1}^{2}}\right)+\frac{(1-w)}{\sigma_{2} \sqrt{2 \pi}} \exp \left(-\frac{\left(x-\mu_{2}\right)^{2}}{2 \sigma_{2}^{2}}\right) \text {, (6) }
$$

where $x=\log e, \mu_{1}$ is the mean of the first peak, $\mu_{2}$ is the mean of the second peak, $\sigma_{1}$ is the variance of the first distribution, $\sigma_{2}$ is the variance of the second distribution, and $w$ is the weight. 

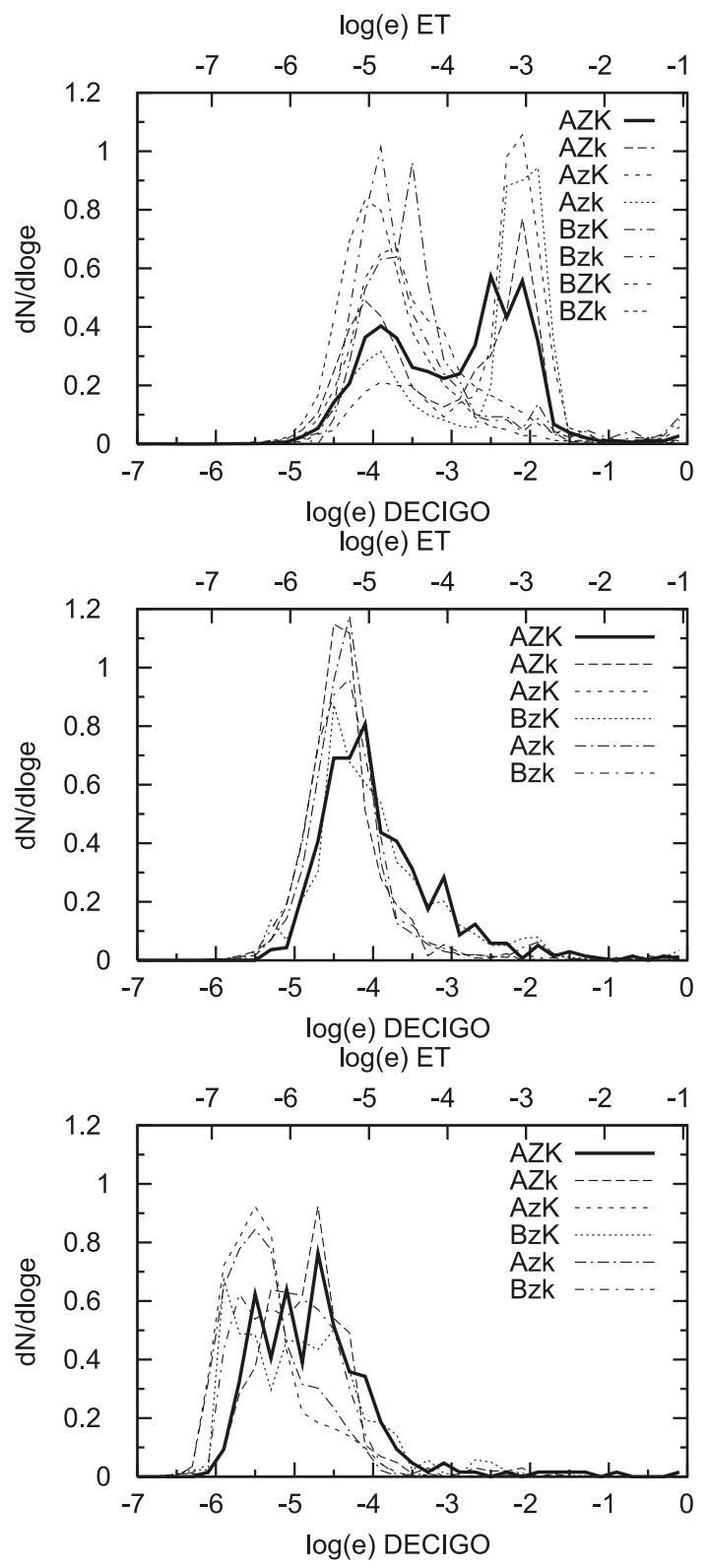

Fig. 5. Distribution of eccentricity for (NS-NS - top panel, BH-NS middle panel and BH-BH bottom panel) seen at $0.3 \mathrm{~Hz}$ (DECIGO - like detectors) and at $3 \mathrm{~Hz}$ (ET - like detectors). Solid thick line corresponds to standard model (AZK). Dashed and dotted lines indicate other models.

We used the Marquardt-Levenberg algorithm to find the parameters, and estimate the asymptotic standard error of each of them. The results of the fits are shown in Table 4. The widths of the distributions $\sigma$ are the same for each frequency band and only the centroids move.

The eccentricity of the $\mathrm{BH}-\mathrm{BH}$ binaries in all three cases is negligible. This is due to two factors. First, at the frequencies of interest the BH-BH systems are much closer to coalescence than the neutron star. Second, the initial kicks at formation of BHs are lower than in the case of NS, so the initial eccentricities of $\mathrm{BH}-\mathrm{BH}$ systems are typically lower than in the case of NS-NS ones.

The eccentricities of the mixed BH-NS systems are larger than in the case of $\mathrm{BH}-\mathrm{BH}$ ones. However, the number of systems is small enough to ensure that by neglecting the eccentricity we do not decrease the sensitivity of Advanced LIGO/VIRGO detectors. For the ET-like detector, some eccentric systems may
Table 4. Parameters of log normal distribution fitted to results of model AZK with asymptotic standard errors.

\begin{tabular}{|c|c|c|c|}
\hline \multicolumn{4}{|c|}{ NS-NS } \\
\hline & $\sigma_{1}:$ & $0.47 \pm 0.04$ & \\
\hline & $\sigma_{2}:$ & $0.39 \pm 0.03$ & \\
\hline & $w:$ & $0.46 \pm 0.03$ & \\
\hline & $0.3 \mathrm{~Hz}$ & $3 \mathrm{~Hz}$ & $30 \mathrm{~Hz}$ \\
\hline$\mu_{1}$ & $-3.83 \pm 0.04$ & $-4.89 \pm 0.04$ & $-5.94 \pm 0.04$ \\
\hline$\mu_{2}$ & $-2.32 \pm 0.03$ & $-3.38 \pm 0.03$ & $-4.43 \pm 0.03$ \\
\hline \multicolumn{4}{|c|}{ BH-NS } \\
\hline \multicolumn{4}{|c|}{$0.55 \pm 0.03$} \\
\hline & $0.3 \mathrm{~Hz}$ & $3 \mathrm{~Hz}$ & $30 \mathrm{~Hz}$ \\
\hline$\mu$ & $-4.18 \pm 0.04$ & $-5.23 \pm 0.03$ & $-6.27 \pm 0.03$ \\
\hline \multicolumn{4}{|c|}{$\overline{\overline{\mathrm{BH}}-\mathrm{BH}}$} \\
\hline \multicolumn{4}{|c|}{$0.70 \pm 0.03$} \\
\hline & $0.3 \mathrm{~Hz}$ & $3 \mathrm{~Hz}$ & $30 \mathrm{~Hz}$ \\
\hline$\mu$ & $-4.91 \pm 0.08$ & $-5.95 \pm 0.03$ & $-7.06 \pm 0.03$ \\
\hline
\end{tabular}

be detected. In the case of the DECIGO-like detector, the number of systems with eccentricities above 0.01 lies between $3 \%$ and $4 \%$.

The eccentricity of NS-NS systems are larger than those of binaries containing BHs. Given a much larger expected detection rate for ET, this means that there should be a significant number of NS-NS binaries with detectable eccentricities. Finally, in the case of DECIGO a fraction of between $2 \%$ and $27 \%$ of the NSNS binaries have eccentricities above 0.01. Moreover, the shape of the eccentricity distribution of NS-NS binaries will depend on the existence of an evolutionary scenario leading to the formation of ultra-compact binaries. Thus, the measurement of the eccentricity distribution is an interesting tool for probing the details of NS-NS formation scenarios.

Acknowledgements. This work was supported by the EGO-DIR-102-2007; the FOCUS 4/2007 Program of Foundation for Polish Science, the Polish grants N N203 511238, DPN/N176/VIRGO/2009, N N203 302835, N N203 404939 and by CompStar a Research Networking Programme of the European Science Foundation.

\section{References}

Abadie, J., Abbott, B. P., Abbott, R., et al. 2010, Classical and Quantum Gravity, 27, 173001

Acernese, F., Amico, P., Al-Shourbagy, M., et al. 2006, Classical and Quantum Gravity, 23, 63

Anderson, S. B., Gorham, P. W., Kulkarni, S. R., Prince, T. A., \& Wolszczan, A. 1990, Nature, 346, 42

Belczynski, K., Bulik, T., \& Kalogera, V. 2002a, ApJ, 571, L147

Belczynski, K., Kalogera, V., \& Bulik, T. 2002b, ApJ, 572, 407

Belczynski, K., Taam, R. E., Kalogera, V., Rasio, F. A., \& Bulik, T. 2007, ApJ, 662,504

Belczynski, K., Kalogera, V., Rasio, F. A., et al. 2008, ApJS, 174, 223

Belczynski, K., Bulik, T., Fryer, C. L., et al. 2010a, ApJ, 714, 1217

Belczynski, K., Dominik, M., Bulik, T., et al. 2010b, ApJ, 715, L138

Bogomazov, A. I., Lipunov, V. M., \& Tutukov, A. V. 2007, Astron. Rep., 51, 308

Burgay, M., D’Amico, N., Possenti, A., et al. 2003, Nature, 426, 531

De Donder, E., \& Vanbeveren, D. 2004, New A, 9, 1

Dessart, L., Burrows, A., Ott, C. D., et al. 2006, ApJ, 644, 1063

Dewi, J. D. M., Pols, O. R., Savonije, G. J., \& van den Heuvel, E. P. J. 2002, MNRAS, 331, 1027

Dewi, J. D. M., Podsiadlowski, P., \& Pols, O. R. 2005, MNRAS, 363, L71

Faulkner, A. J., Kramer, M., Lyne, A. G., et al. 2005, ApJ, 618, L119

Fryer, C. L., \& Kalogera, V. 2001, ApJ, 554, 548

Gondek-Rosińska, D., Bulik, T., \& Belczyński, K. 2007, Adv. Space Res., 39, 285

Harry, G. M., \& the LIGO Scientific Collaboration. 2010, Classical and Quantum Gravity, 27, 084006

Hobbs, G., Lorimer, D. R., Lyne, A. G., \& Kramer, M. 2005, MNRAS, 360, 974 
I. Kowalska et al.: The eccentricity distribution of compact binaries

Hut, P. 1981, A\&A, 99, 126

Ivanova, N., \& Taam, R. E. 2004, ApJ, 601, 1058

Kawamura, S. 2006, Astronomical Herald, 99, 490

Kiel, P. D., Hurley, J. R., \& Bailes, M. 2010, MNRAS, 406, 656

Kitaura, F. S., Janka, H., \& Hillebrandt, W. 2006, A\&A, 450, 345

Lorimer, D. R., Stairs, I. H., Freire, P. C., et al. 2006, ApJ, 640, 428

Mandel, I., \& O'Shaughnessy, R. 2010, Classical and Quantum Gravity, 27, 114007

Nelemans, G., \& van den Heuvel, E. P. J. 2001, A\&A, 376, 950

Peters, P. C. 1964, Phys. Rev., 136, 1224

Peters, P. C., \& Mathews, J. 1963, Phys. Rev., 131, 435

Pfahl, E., Podsiadlowski, P., \& Rappaport, S. 2005, ApJ, 628, 343

Schnittman, J. D. 2004, Phys. Rev. D, 70, 124020

Seto, N., Kawamura, S., \& Nakamura, T. 2001, Phys. Rev. Lett., 87, 221103
Shapiro Key, J., \& Cornish, N. J. 2010 [arXiv: 1006. 3759]

Sipior, M. S., \& Sigurdsson, S. 2002, ApJ, 572, 962

Smith, J. R., \& LIGO Scientific Collaboration 2009, Classical and Quantum Gravity, 26, 114013

Spallicci, A. D. A. M., Aoudia, S., de Freitas Pacheco, J., Regimbau, T., \& Frossati, G. 2005, Classical and Quantum Gravity, 22, 461

Taam, R. E., \& Sandquist, E. L. 2000, ARA\&A, 38, 113

Timmes, F. X., Woosley, S. E., \& Weaver, T. A. 1996, ApJ, 457, 834

Van Den Broeck, C. 2010 [arXiv: 1003.1386]

Voss, R., \& Tauris, T. M. 2003, MNRAS, 342, 1169

Webbink, R. F. 1984, ApJ, 277, 355

Weisberg, J. M., \& Taylor, J. H. 2005, in Binary Radio Pulsars, ed. F. A. Rasio, \& I. H. Stairs, ASP Conf. Ser., 328, 25

Wolszczan, A. 1991, Nature, 350, 688 